\title{
CSF1R-dependent myeloid cells are required for NK-mediated control of metastasis
}

\author{
Michal Beffinger, ${ }^{1}$ Paulino Tallón de Lara, ${ }^{1}$ Sònia Tugues, ${ }^{1}$ Marijne Vermeer, ${ }^{1}$ Yannick Montagnolo, ${ }^{1}$ \\ Isabel Ohs, ${ }^{1}$ Virginia Cecconi, ${ }^{1}$ Giulia Lucchiari, ${ }^{1}$ Aron Gagliardi, ${ }^{1}$ Nikola Misljencevic, ${ }^{1}$ James Sutton, ${ }^{2}$ \\ Roman Spörri,, ${ }^{3}$ Burkhard Becher, ${ }^{1}$ Anurag Gupta, ${ }^{1}$ and Maries van den Broek ${ }^{1}$ \\ Institute of Experimental Immunology, University of Zurich, Zurich, Switzerland. ${ }^{2}$ Novartis Institutes for Biomedical \\ Research, Emeryville, California, USA. ${ }^{3}$ Institute of Microbiology, ETH Zurich, Zurich, Switzerland.
}

Myeloid leukocytes are essentially involved in both tumor progression and control. We show that neo-adjuvant treatment of mice with an inhibitor of CSF1 receptor (CSF1R), a drug that is used to deplete tumor-associated macrophages, unexpectedly promoted metastasis. CSF1R blockade indirectly diminished the number of NK cells due to a paucity of myeloid cells that provide the survival factor IL-15 to NK cells. Reduction of the number of NK cells resulted in increased seeding of metastatic tumor cells to the lungs but did not impact on progression of established metastases. Supplementation of mice treated with CSF1R-inhibitor with IL-15 restored numbers of NK cells and diminished metastasis. Our data suggest that CSF1R blockade should be combined with administration of IL-15 to reduce the risk of metastasis.

Authorship note: MB and PTDL contributed equally to this work.

Conflict of interest: |S was an employee of Novartis International AG at the time when the experiments were performed.

Submitted: September 29, 2017

Accepted: April 13, 2018

Published: May 17, 2018

\section{Reference information:} JCI Insight. 2018;3(10):e97792. https://doi.org/10.1172/jci. insight.97792.

\section{Introduction}

Despite recent advances in oncology, metastatic disease remains the major cause of cancer-related death. The cascade of events involved in this process includes invasion of surrounding tissue, intravasation into the blood or lymph vasculature, survival in the circulation, and extravasation into and colonization of distant tissue. Each of these steps involves crosstalk between tumor cells and hematopoietic cells, including lymphocytes and different cell types of myeloid origin (1).

NK cells primarily control circulating tumor cells (2), while $\mathrm{CD}^{+} \mathrm{T}$ cells can control transformed cells in primary tumors, as well as in metastatic lesions (3). $\mathrm{CD}^{+} \mathrm{T}$ cells became an attractive target for immune-activating therapies $(4,5)$, and approaches to boost NK cell responses are under development (6). Progress in therapeutic strategies targeting the myeloid compartment has proven more complicated due to the dual role these cells play in tumor progression and metastasis. On the one hand, myeloid cells can produce proinflammatory cytokines and survival factors for NK and T cells, such as IL-2, IL-12, and IL-15 (7-9), and comprise antigen-presenting cells (APCs) that are crucial to T cell activation (10). On the other hand, they are recruited to tumors, where they contribute to local immunosuppression, promote tumor growth, and directly facilitate formation of metastases (11-13). Targeting tumor-associated macrophages (TAMs) by inhibition of CSF1R has shown promising results in preclinical cancer models (14-17) and in diffuse-type tenosynovial giant cell tumors, which are driven by CSF1 in an autocrine manner (18). Data on CSF1R blockade in malignant disease including metastasis are largely pending (19). However, little is known about the impact of such treatment on the formation or progression of metastasis. Here, we aimed to answer this question and investigated the impact of CSF1R blockade on metastasis when given as a monotherapy in a neo-adjuvant or adjuvant setting.

\section{Results}

Targeting CSF1R ${ }^{+}$myeloid cells promotes metastatic disease. We used a model of spontaneous metastasis from resected, primary Lewis lung carcinoma tumors expressing luciferase (LLC-LUC) in immunocompetent mice. Within 3 weeks after tumor resection, approximately $50 \%-70 \%$ of mice develop metastases preferentially in the lungs, liver, or tumor-draining lymph nodes as detected by bioluminescent imaging. Macrophages, defined as $\mathrm{CD} 45.2^{+} \mathrm{CD} 11 \mathrm{~b}^{+} \mathrm{Ly} 6 \mathrm{G}^{-} \mathrm{F} 4 / 80^{+} \mathrm{Ly} 6 \mathrm{C}^{\text {int }}$ cells, build the majority of tumor-infiltrating leukocytes (Supplemental Figure 1; supplemental material available online with 
A

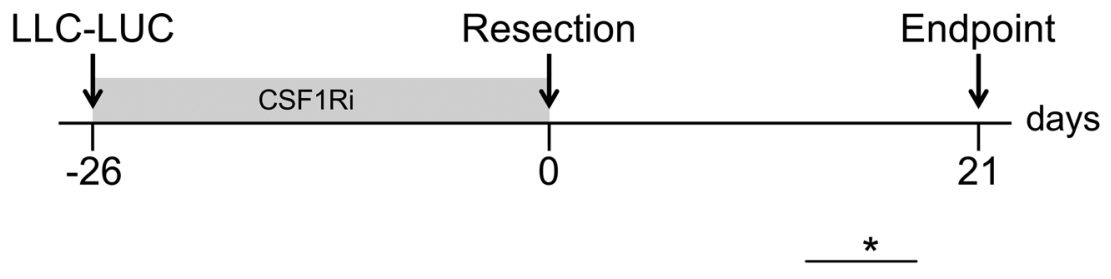

B

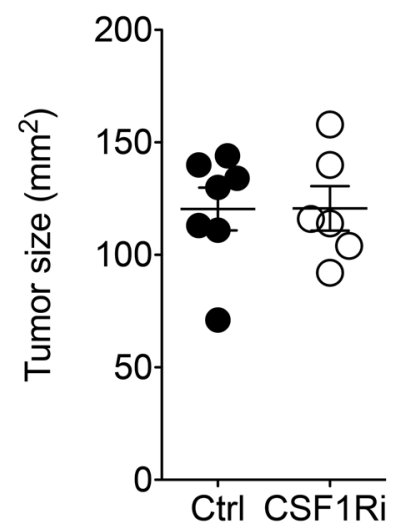

C

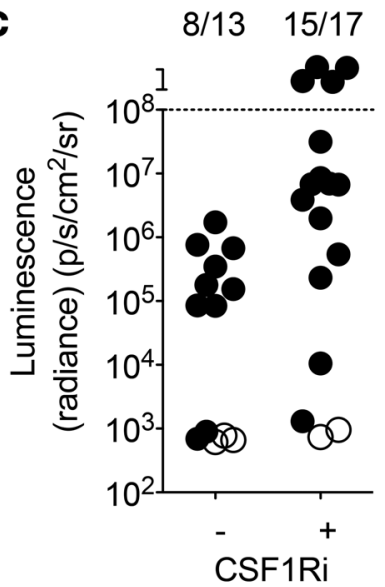

\section{4T1-LUC}

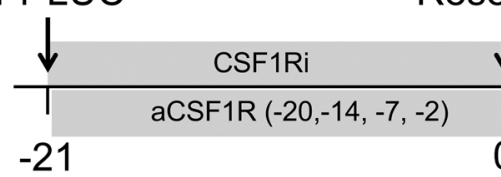

Endpoint days

14
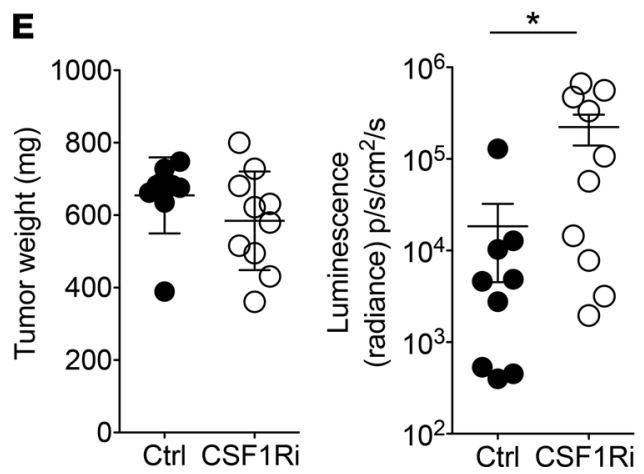

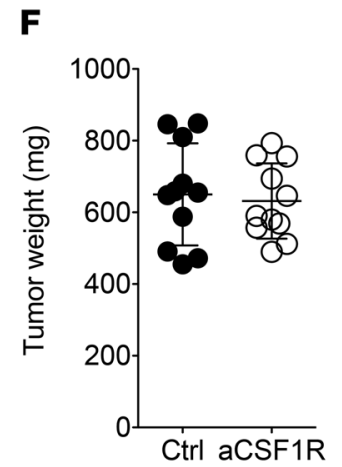

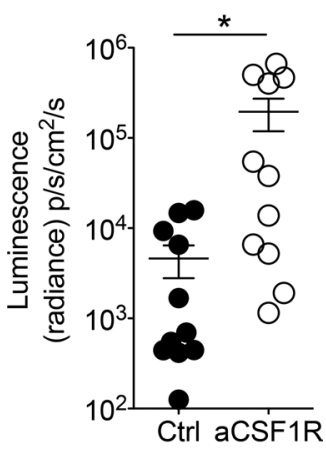

Figure 1. Blocking CSF1R increases the risk of developing metastatic disease. (A-C) Spontaneous metastasis from s.c. LLC-LUC tumors in C57BL/6 mice. (A) Experimental timeline. (B) Primary tumor size at resection. Mean \pm SD. Each symbol represents an individual mouse (Ctrl, $n=7$; CSF1Ri, $n=6$ ). Representative data from 2 independent experiments are shown. (c) Metastatic burden presented as sum of luminescent signals from lung, liver, and both tumor-draining lymph nodes for each mouse. Filled circles represent mice with detectable metastasis; open circles represent metastasis-free mice. Mice above the dotted line had to be sacrificed before the endpoint due to metastatic burden. Numbers above the graph show number of mice with metastasis in total cohort of animals. ${ }^{*} P<0.05$ $\left(\chi^{2}\right.$ test). Pooled data from 2 independent experiments are shown (Ctrl, $n=13$; CSF1Ri, $n=17$ ). (D-F) Spontaneous lung metastasis from autochthonous 4T1 tumors in BALB/C mice. (D) Experimental timeline. Mice were treated daily with a small-molecule inhibitor of CSF1R (E) or with blocking anti-CSF1R antibody on the indicated days (F). (E and F) Primary tumor weight at resection. Mean \pm SD. Lung metastases quantified by bioluminescence. Mean \pm SEM. ${ }^{*} P$ $<0.05$ (2-tailed Student's $t$ test with Welch's correction). Each symbol represents an individual mouse. (E) Ctrl, $n=9$; CSF1Ri, $n=$ 10. (F) Ctrl and CSF1Ri, $n=11$.

this article; https://doi.org/10.1172/jci.insight.97792DS1) and were shown to play a tumor-promoting role (11-13). All TAMs express CSF1R as well as CD206, Egr2, MHC class II, and low amounts of iNOS (data not shown), suggesting an M2-like phenotype. To address the impact of macrophages on the development of metastasis, we used BLZ945, a small-molecule inhibitor that blocks CSF1R-signaling (CSF1R inhibitor; CSF1Ri) and thus prevents the development and maintenance of various myeloid cell subsets, including CSF1R-expressing TAMs $(16,20)$. In order to investigate the contribution of $\mathrm{CSF} 1 \mathrm{R}^{+}$myeloid cells on the initial stages of the metastatic cascade, we administered CSF1Ri from the time of tumor cell injection until the resection of the primary tumor (neo-adjuvant treatment, Figure 1A). Administration of CSF1Ri did not influence the growth of the primary tumor, as illustrated by the size at resection (Figure 1B). In contrast, a significantly higher proportion of mice treated with neo-adjuvant CSF1Ri developed metastases than control mice treated with the vehicle, $20 \%$ Captisol (Figure 1C). We blocked CSF1R in the 4T1 model of spontaneous metastasis from breast cancer in BALB/c mice using CSF1Ri or antibody-mediated blockade of CSF1R before resection of the primary tumor (Figure 1D) and again observed that CSF1R blockade had no impact on the weight of the primary 
tumor but resulted in increased metastasis to the lungs (Figure 1, E and F). Also, in the context of 4T1 breast cancer, CSF1R blockade significantly reduced the number of circulating NK cells (Supplemental Figure 2, A-C). Our observation that CSF1R blockade does not influence the growth of the primary tumor in different experimental setups is in agreement with recently published findings (21). Because 4T1 cells metastasize to the lungs before resection is required for ethical reasons, we have blocked CSF1R-using antibodies and analyzed subsequent lung metastasis without resection of the primary tumor (Supplemental Figure 3A). Also with continuous presence of the primary tumor - whose size was not affected by CSF1R blockade (Supplemental Figure 3B) - anti-CSF1R resulted in increased metastatic load in the lungs (Supplemental Figure 3C).

As resection of the primary tumor removes the source of circulating, metastasizing tumor cells, which have short half-lives in the circulation (22), we administered CSF1Ri immediately after resection until the endpoint (adjuvant treatment) to investigate the impact of CSF1R ${ }^{+}$cells on metastatic outgrowth and found that adjuvant CSF1Ri had no impact on metastasis (Supplemental Figure 4).

Together, these results suggest that systemic blockade of CSF1R in a neo-adjuvant setting increases the risk of developing metastasis.

Systemic inhibition of CSF1R-signaling affects NK cell homeostasis and promotes metastatic seeding. To understand why CSF1R blockade promotes metastasis, we determined the numbers of tumor-associated and circulating leukocytes in mice treated with CSF1Ri. As expected, treatment of tumor-bearing mice with CSF1Ri reduced the numbers of tumor-associated monocytes and macrophages (Figure 2A and Supplemental Figure 5A), as well as the number of circulating Ly6 $\mathrm{C}^{\text {hi }}$ and Ly6 $\mathrm{C}^{\mathrm{lo}}$ monocytes (Figure $2 \mathrm{~B}$ ). Treatment with CSF1Ri also resulted in decreased numbers of tumor-associated and circulating NK cells, as well as $\mathrm{CD}^{+} \mathrm{T}$ cells (Figure 2, $\mathrm{A}$ and $\mathrm{B}$ ), whereas the numbers of neutrophils, B cells, and $\mathrm{CD}^{+} \mathrm{T}$ cells were not affected (Figure $2 \mathrm{~B}$ ). This was independent of the presence of a tumor, since treatment with CSF1Ri in non-tumor-bearing mice also significantly reduced the number of circulating Ly $6 \mathrm{C}^{\text {hi }}$ and Ly6 $\mathrm{C}^{\text {lo }}$ myeloid and NK cells, and showed a tendency of less CD8 ${ }^{+}$cells (Supplemental Figure 5B). CSF1Ri result-

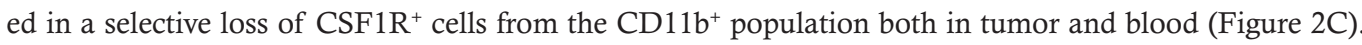
The apparent discrepancy regarding $\mathrm{NK}$ and $\mathrm{CD} 8^{+} \mathrm{T}$ cell numbers between Figure 2 and Supplemental Figure 3B may be caused by the fact that we treated mice with CSF1Ri for 7 days in Figure 2 and only for 5 days in Supplemental Figure 5B; furthermore, this can be explained by variation between experiments, mainly because of sample processing. In fact, the percentage of $\mathrm{CD}^{+}$as well as the percentage of NK cells of live $\mathrm{CD} 45^{+} \mathrm{Lin}^{-}$cells in the control group was similar in both experiments (data not shown). In contrast, CSF1Ri did not show a measurable effect on the number of circulating neutrophils, $\mathrm{CD}^{+} \mathrm{T}$ cells, or B cells (Supplemental Figure 5C). Treatment with a CSF1R-blocking antibody induced similar changes in the number of circulating total, Ly6 $\mathrm{C}^{\text {hi }}$, and Ly6 $\mathrm{C}^{\text {lo }}$ monocytes, as well as NK cells (Supplemental Figure 5D), but did not affect the number of $\mathrm{CD}^{+} \mathrm{T}$ cells (not shown).

In addition to depleting monocytic cells, CSF1R blockade also affects the number of CD8 ${ }^{+} \mathrm{T}$ and NK cells; therefore, we investigated the contribution of those effector cells to controlling metastasis. As CSF1Ri did not promote metastasis when the treatment was started after resection (Supplemental Figure 4), we speculated that $\mathrm{CD}^{+} \mathrm{T}$ or NK cells control the seeding of circulating tumor cells. For this reason, we used i.v.-injected tumor cells as a model, as it eliminates potential effects of the primary tumor other than providing metastasizing tumor cells. To study whether T cells play a role in controlling metastatic seeding, we depleted $\mathrm{CD}^{+}$or $\mathrm{CD}^{+}$cells prior to i.v. injection of LLC-LUC cells and compared the metastatic load in the lungs 1 day later. We observed a similar tumor cell load in the lungs in all groups (Figure 3A, left panel) and concluded that $\mathrm{T}$ cells play no detectable role in the metastatic seeding of circulating tumor cells to the lungs. To exclude a role for $\mathrm{CD} 8^{+} \mathrm{T}$ cells in controlling metastatic outgrowth, we compared the metastatic load in the lungs between $\mathrm{CD}^{+}$-depleted and control mice 16 days after i.v. injection and observed no difference (Figure 3A, right panel). A single injection of anti-CD8 resulted in depletion of circulating $\mathrm{CD} 8^{+} \mathrm{T}$ cells for at least 18 days (Supplemental Figure 6). Collectively, these results exclude a role for $\mathrm{CD}^{+} \mathrm{T}$ cells in controlling metastatic seeding and outgrowth.

We therefore speculated that the increased proportion of mice with metastasis after CSF1Ri treatment is the consequence of the concomitant loss of NK cells. To directly address whether NK cells mainly eliminate circulating tumor cells or also interfere after metastatic seeding has occurred, we depleted NK cells prior to or 24 hours after i.v. injection of LLC-LUC cells and compared the metastatic load in the lungs 14 days later. Tumor cells extravasate and are cleared from the circulation within 24 hours after i.v. 
A

LLC-LUC

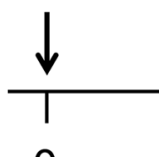

0
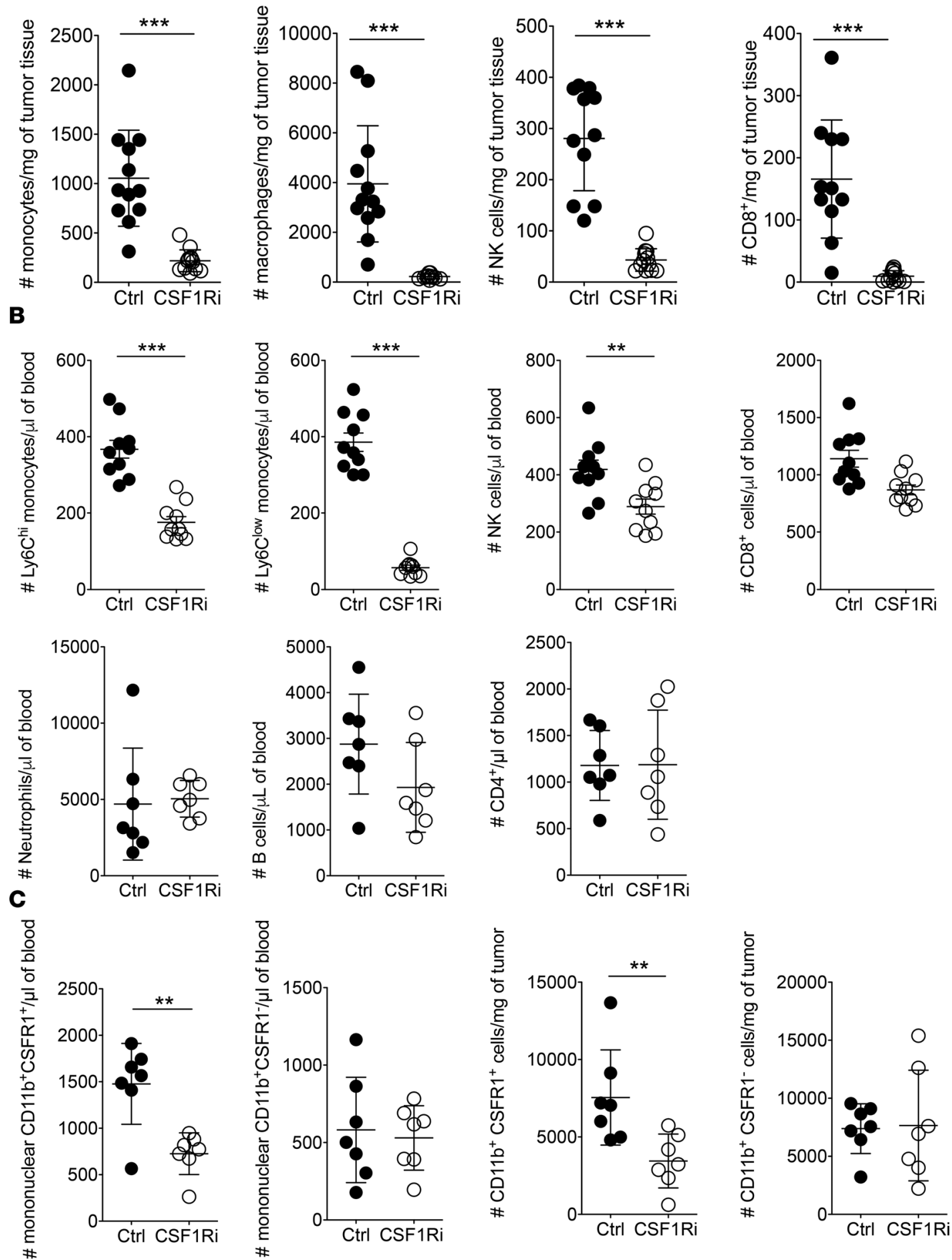

Figure 2. Administration of CSF1Ri results in concomitant loss of NK cells. Administration of CSF1Ri starting 8 days before resection results in loss of NK, CD8+, and myeloid cells in the tumor (A) and blood (B) as measured by flow cytometry. Ly6C hi and Ly6C ${ }^{10}$ cells represent inflammatory and patrolling monocytes, respectively. Monocytes, CD45.2+CD11b+CSF1R+ NK cells, CD45.2+CD3-NK1.1 + CD8 ${ }^{+}$T cells, CD45.2 $2^{+}$CD3 $3^{+}$CD8 $\alpha^{+}$; neutrophils, CD45.2+CD11 b+Ly6C'; B cells, CD45.2+CD19+; CD4 ${ }^{+} T$ cells, CD45.2+CD3+CD4+. Analysis was performed after gating on live singlets. (C) Administration of CSF1Ri starting 8 days before resection results in selective loss of CSF1R ${ }^{+}$cells from the CD11b+ population in the blood (left panels) and tumor (right 
panels). CSF1R expression was visualized using CSF1R-reporter mice (MacGreen). (A-C) Each symbol represents an individual mouse. (A) Ctrl and CSF1Ri, $n=11$. (B and C) Ctrl and CSF1Ri, $n=7$. Mean \pm SD. ${ }^{*} P<0.01,{ }^{* *} P<0.005$ (2-tailed Student's $t$ test with Welch's correction). A representative experiment of 2 is shown.

injection (2). Depletion of NK cells before i.v. injection resulted in significantly increased tumor burden in the lungs, whereas depletion after i.v. injection had no impact on metastasis (Figure 3B). The increased tumor burden in the lung as a result of prior NK depletion was already apparent as early as 20 minutes after i.v. injection of LLC-LUC cells (Figure 3C, left panel). Although the tumor load decreased between 20 minutes and 24 hours after injection of tumor cells independently of NK cell depletion, the NK celldependent difference remained (Figure 3C).

NK cells also control spontaneous metastasis, since depletion of NK cells shortly before surgery resulted in a significantly higher proportion of mice with metastasis (Figure 3D).

Finally, to determine whether CSF1Ri-induced reduction of NK cell numbers explains the increased metastatic burden that we observed after CSF1Ri administration, we directly compared metastatic seeding in mice that were treated for 3 days with CSF1Ri or were depleted of NK cells using anti-NK1.1. Both treatments significantly reduced the number of circulating NK cells. Whereas no NK cells were detectable after anti-NK1.1 treatment, we still detected NK cells in CSF1Ri-treated mice (Figure 3E, left panel). Both treatments significantly increased metastatic seeding, but NK-depleted mice showed higher metastatic burden than CSF1Ri-treated mice (Figure 3E, right panel), suggesting a correlation between circulating NK cell counts and protection against metastatic seeding.

Together, these results indicate that NK cells - and not T cells - are essential to prevent metastatic seeding but are dispensable for metastatic outgrowth of seeded tumor cells.

Administration of exogenous IL-15 during CSF1Ri treatment restores NK cell numbers and metastasis control. We observed that CSF1R blockade results in an expected reduction of CSF1R-dependent myeloid cells, as well as in a rather unexpected loss of $\mathrm{NK}$ and $\mathrm{CD} 8^{+} \mathrm{T}$ cells. Homeostasis of $\mathrm{NK}$ and $\mathrm{CD} 8^{+} \mathrm{T}$ cells depends on cross-presentation of IL-15 by IL-15R $\alpha$-expressing cells. Because IL-15R $\alpha$ is expressed by CSF1R positive cells, we reasoned that losing such cells reduces the amount of IL-15 available to CD8 ${ }^{+} \mathrm{T}$ and NK cells (23-27). Because the concentration of IL-15 in vivo is too low to detect and expression of the transpresenting IL-15R $\alpha$ chain under steady state conditions is virtually undetectable (27), we performed an IL-15 transpresentation assay using phosphorylation of Stat5 (pStat5) in NK cells as a readout for signaling via the IL-15R. We observed that both $\mathrm{CD} 11 \mathrm{~b}^{+} \mathrm{CD} 115^{+}$and $\mathrm{CD} 11 \mathrm{~b}^{+} \mathrm{CD} 115^{-}$induced pStat5 in NK cells. This was dependent on transpresented IL-15, as we detected virtually no pSTAT5 in NK cells that were incubated with $\mathrm{CD} 11 \mathrm{~b}^{+} \mathrm{CD} 115^{+}$or $\mathrm{CD} 11 \mathrm{~b}^{+} \mathrm{CD} 115^{-}$cells sorted from $\mathrm{Il15 \textrm {ra } ^ { - / }}$ mice (Supplemental Figure 7). In mice treated with CSF1Ri or anti-CSF1R, CD11b $\mathrm{b}^{+} \mathrm{CD} 115^{+}$myeloid cells - which make up a substantial proportion of the cells that transpresent IL-15 - are lost. Consequently, we suggest that the reduction of NK cells could be a result of diminished transpresentation of IL-15.

To address whether restoration of NK cell numbers during treatment with CSF1Ri reverts increased metastasis, we treated mice with CSF1Ri and supplemented 1 group with IL-15/IL-15R $\alpha$ complexes (IL-15c) (Figure 4A), thus rescuing NK cell numbers in the face of low numbers of CSF1R-dependent myeloid cells. Control groups were treated with vehicle or vehicle plus IL-15c. The numbers of CSF1R-dependent myeloid cells remained low in CSF1Ri-treated mice supplemented with IL-15c (Supplemental Figure 8A) $(28,29)$. CSF1Ri-treated mice showed strongly reduced NK cell counts, whereas CSF1Ri-treated mice that received exogenous IL-15c or mice that received IL-15c plus vehicle showed supraphysiological numbers of NK cells at the time point of i.v. injection of tumor cells (Figure 4B). Decreased numbers of NK cells correlated with increased metastatic load in the lungs, whereas IL-15ctreated mice developed less metastasis (Figure 4, C and D).

We investigated whether the administration of IL-15c influenced NK cell maturation or activation. Exogenously administered IL-15c did not change the expression of CD69 or FasL (CD95) by NK cells, but it led to a slightly reduced the expression of DNAM1 (CD226) and an increased expression of KLRG1 (Supplemental Figure $8 \mathrm{~B})$. Furthermore, we found a higher proportion of less differentiated $\left(\mathrm{CD} 27^{+} \mathrm{CD} 11 \mathrm{~b}^{+}\right)$ and a lower proportion of terminally differentiated $\left(\mathrm{CD} 27^{-} \mathrm{CD} 11 \mathrm{~b}^{+}\right) \mathrm{NK}$ cells in mice that received IL-15c (Supplemental Figure 8C) The relevance of these findings in the context of protection against metastatic seeding is difficult to interpret at this point because both $\mathrm{CD} 27^{+} \mathrm{CD} 11 \mathrm{~b}^{+}$and $\mathrm{CD} 27^{-} \mathrm{CD} 11 \mathrm{~b}^{+} \mathrm{NK}$ cells can execute this function $(30,31)$. 
A

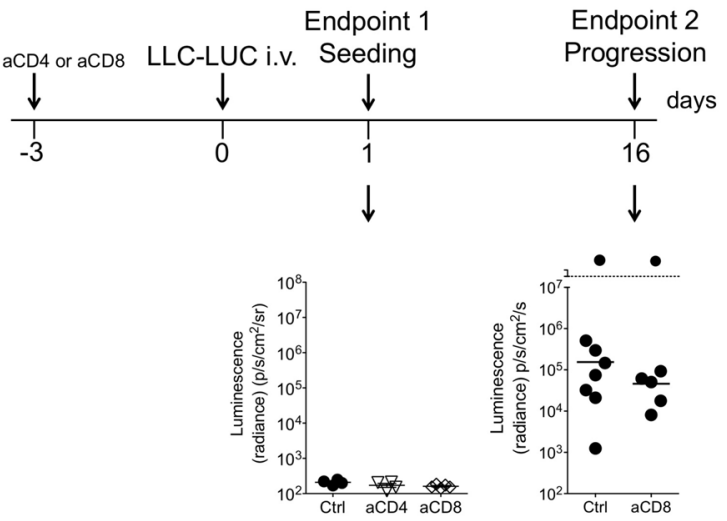

$\mathbf{C}_{\text {a }}$
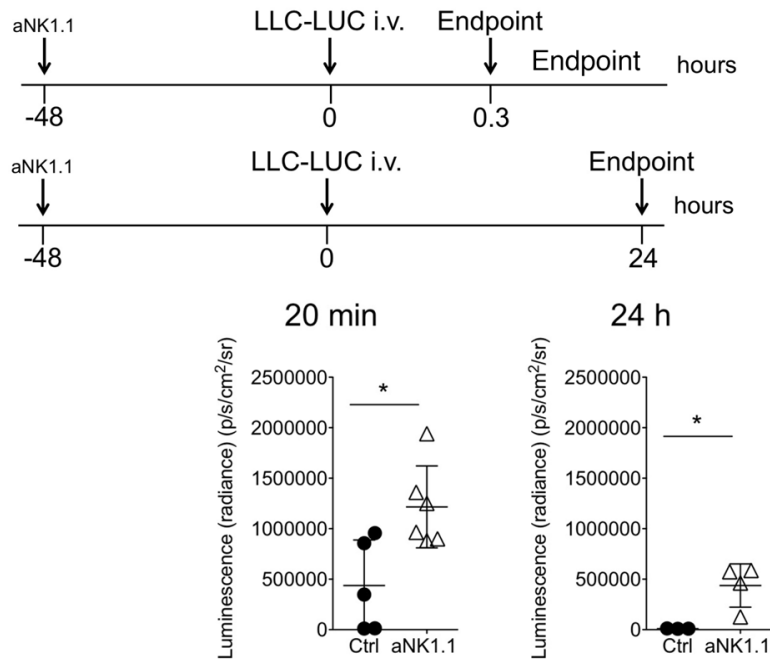

B
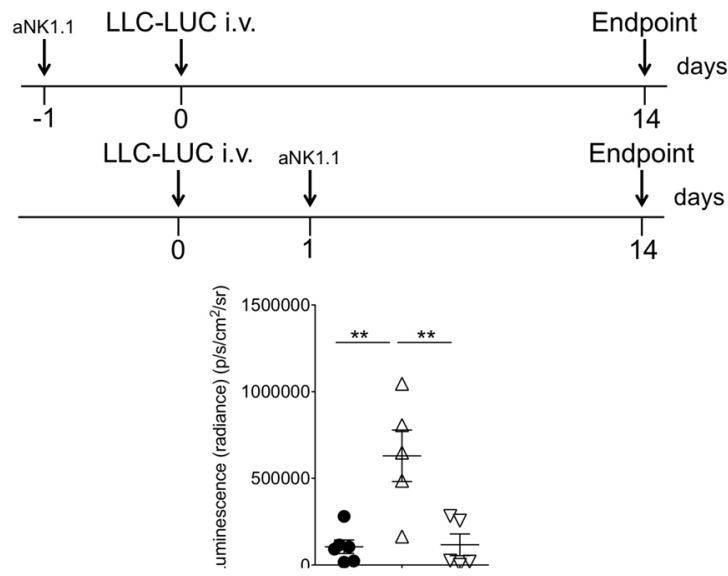

D
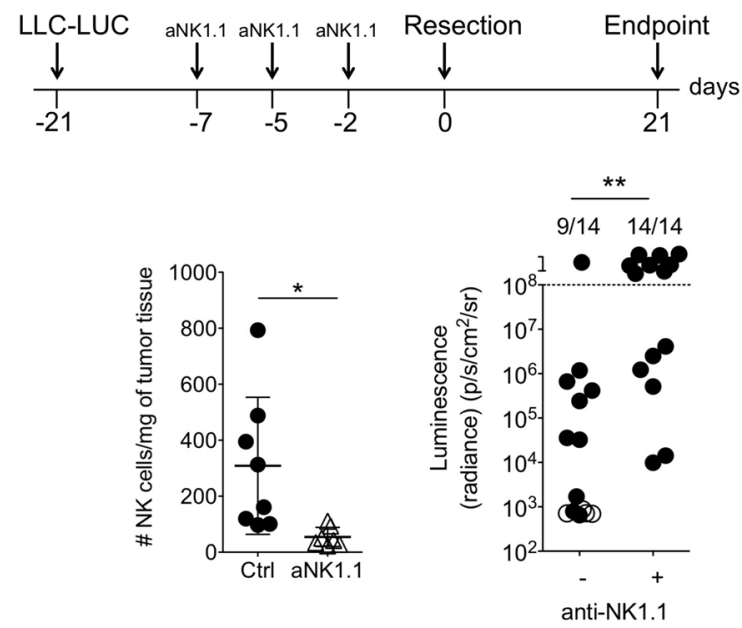

$\mathbf{E}$

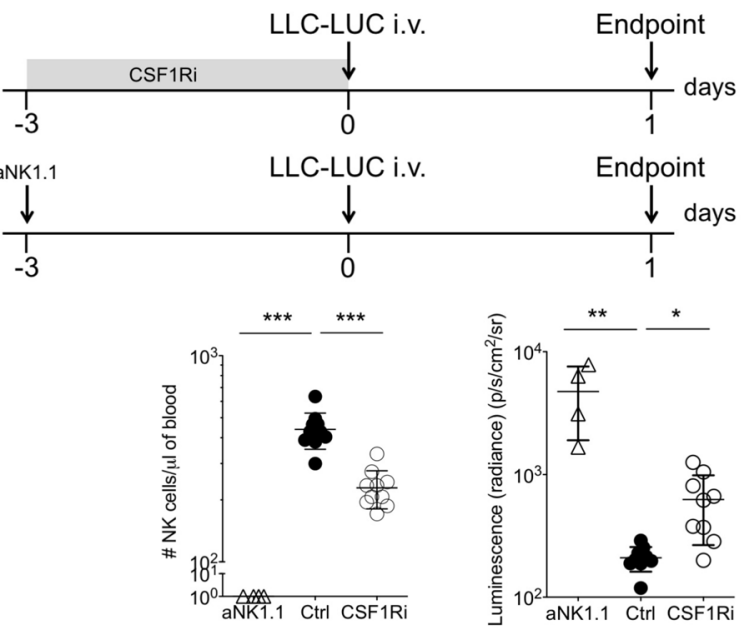

Figure 3. Administration of CSF1Ri promotes metastasis via concomitant loss of NK cells. (A) LLC-LUC cells were i.v. injected into C57BL/6 mice that were depleted of $\mathrm{CD} 4^{+}$or $\mathrm{CD}^{+}$cells. The metastatic burden was determined 24 hours (endpoint 1) or 16 days (endpoint 2) after tumor cell injection by bioluminescent imaging on resected lungs. Each symbol represents an individual mouse. Endpoint 1, Ctrl and aCD4, $n=4 ;$ aCD8, $n=5$. Endpoint 2, Ctrl, $n=8 ; a C D 8, n=6$. Mean \pm SD. Groups are not statistically significantly different (1-way ANOVA with Bonferroni's correction). (B) LLC-LUC cells were i.v. injected into C57BL/ 6 mice that were depleted of NK cells immediately before or afterward. The metastatic burden was determined 14 days after tumor cell injection by bioluminescent imaging on resected lungs. Each symbol represents an individual mouse. Ctrl and +24 hours, $n=6 ;-24$ hours, $n=5$. Mean \pm SD. ${ }^{* *} P<0.01$ (one-way ANOVA with Bonferroni's correction). (C) LLC-LUC cells were i.v. injected into NK cell-depleted C57BL/6 mice. The metastatic burden was determined 20 minutes and 1 hour after tumor cell injection by bioluminescent imaging on resected lungs. Each symbol represents an individual mouse. Twenty minutes, Ctrl, $n=5$; aNK1.1, $n=6$. Twenty-four hours, Ctrl, $n=3$; aNK1.1, $n=4$. Mean \pm SD. ${ }^{*} P<0.05$ ( 2 -tailed Student's $t$ test 
with Welch's correction). (D) Mice bearing s.c. LLC-LUC tumors were depleted from NK cells before resection. Lower left panel: Number of NK cells in the resected tumor. Each symbol represents an individual mouse. Ctrl and aNK1.1, $n=8$. Mean \pm SD. ${ }^{*} P<0.05$ (2-tailed Student's $t$ test with Welch's correction). Lower right panel: Spontaneous metastasis to the lungs is presented as sum of luminescent signals from lung, liver, and both tumor-draining lymph nodes for each mouse. Filled circles represent mice with detectable metastasis; open circles represent metastasis-free mice. Mice above the dotted line had to be sacrifice before the endpoint due to metastatic burden. Numbers above the graph show number of mice with metastasis in total cohort of animals (Ctrl and aNK.1.1, $n=14$ ). ${ }^{* *} P<0.01\left(\chi^{2}\right.$ test). (E) Metastatic seeding to the lungs measured 1 day after i.v. injection of LLC-LUC cells into NK cell-depleted, CSF1Ri-treated, or control mice. The left panel shows the number of circulating NK cells at day 0 . The right panel shows the metastatic burden to the lungs measured by bioluminescent imaging on resected lungs. Each symbol represents an individual mouse. Ctrl, $n=9$; aNK.1.1, $n=4$; CSF1Ri, $n=$ 9. Mean \pm SD. ${ }^{*} P<0.05,{ }^{*} P<0.01,{ }^{* *} P<0.005$ (1-way ANOVA with Bonferroni's correction). A representative experiment of 2 is shown.

Because the number of $\mathrm{CD} 8^{+} \mathrm{T}$ cells also depends on transpresented IL-15, we investigated a possible contribution of $\mathrm{CD}^{+} \mathrm{T}$ cells to the IL-15c-mediated protection against metastasis promoted by blocked CSF1R. Therefore, we administered IL-15c to CSF1Ri-treated mice that were or were not depleted of $\mathrm{CD}^{+} \mathrm{T}$ cells. We observed that IL-15c protected against CSF1Ri-induced metastasis independently of $\mathrm{CD}^{+} \mathrm{T}$ cells (Supplemental Figure 9).

Finally, to further strengthen the point that the number of circulating NK cells is a major factor influencing the metastatic load in the lungs, we directly corrected the number of circulating $\mathrm{NK}$ in CSF1Ri-treated mice by adoptively transferring sorted NK cells. Adoptively transferred NK cells reduced the metastatic load in CSF1Ri-treated mice to that in untreated mice (Figure 5).

Thus, we conclude that CSF1Ri blockade induces a loss of NK cells secondary to paucity of IL-15 transpresenting myeloid cells, which promotes metastasis to the lungs.

\section{Discussion}

Tumor-associated, CSF1R-dependent macrophages promote tumor progression, restrict antitumor immunity, and support tumor cells during all stages of the metastatic cascade $(13,32)$. This is further underlined by the observation that tumors often sustain macrophage differentiation and survival by producing CSF1 (33-35). CSF1R has, thus, become an attractive therapeutic target, and CSF1R blockade has yielded promising results in preclinical and clinical studies (14-16, 18, 36, 37). For example, CSF1R blockade retards the development of primary tumors and promotes $\mathrm{CD} 8^{+} \mathrm{T}$ cell-mediated antitumor immunity $(14,16,38)$. Furthermore, CSF1R inhibition synergizes with checkpoint blockade therapy in controlling primary tumor lesions $(15,39)$.

Because most studies have focused on how CSF1R blockade influences progression of primary tumors, little is known about the effect of CSF1R blockade on metastasis. We addressed this question using clinically relevant models for spontaneous metastasis. We applied CSF1R blockade before (neo-adjuvant) or after (adjuvant) resection of the primary tumor. Neo-adjuvant CSF1R blockade had no impact on the progression of primary tumors but unexpectedly promoted metastasis, whereas adjuvant treatment had no effect on metastasis.

One other study previously reported the metastasis-promoting effect of CSF1R blockade (40). The authors showed that CSF1R blockade in tumor-bearing mice results in an increased concentration of granulocyte CSF (G-CSF) in the serum and, thus, proposed G-CSF-induced neutrophilia as the main cause of increased metastasis (40), although they did not experimentally test this hypothesis. Neutrophilia is observed in many preclinical cancer models, including 4T1 and LLC, and is a consequence of tumor-derived G-CSF $(2,41,42)$. A further increase of neutrophil counts downstream of CSF1R blockade, however, may be a peculiarity of certain tumors, as we did not observe a BLZ945-induced increase of neutrophil counts in tumor-bearing (LLC-LUC) or naive mice upon treatment with BLZ945. Also, blocking CCL2 increases the risk of developing metastasis upon treatment cessation due to enhanced angiogenesis and monocyte counts (43). Thus, targeting myeloid cells in cancer may be less straightforward than thought, and therefore, a better understanding of the role of different myeloid subsets in various aspects of cancer development and progression is urgently needed.

Here, we offer a mechanistic explanation for the apparently controversial effects of CSF1R blockade in the context of cancer $(14,16,39,40)$ by discovering that treatment with CSF1Ri concomitantly reduces NK cell counts. Maintenance of peripheral NK cells crucially depends on IL-15, as IL-15R signaling controls the intracellular levels of antiapoptotic Bcl-2 (44). NK cells express IL-15R $\beta / \gamma$, which binds IL-15 with high affinity only when it is transpresented on IL-15R $\alpha$ expressed by myeloid cells $(23,24,26)$. We observed that the detrimental effect of CSF1R blockade on metastasis is reversed by exogenous IL-15c (28, 
A
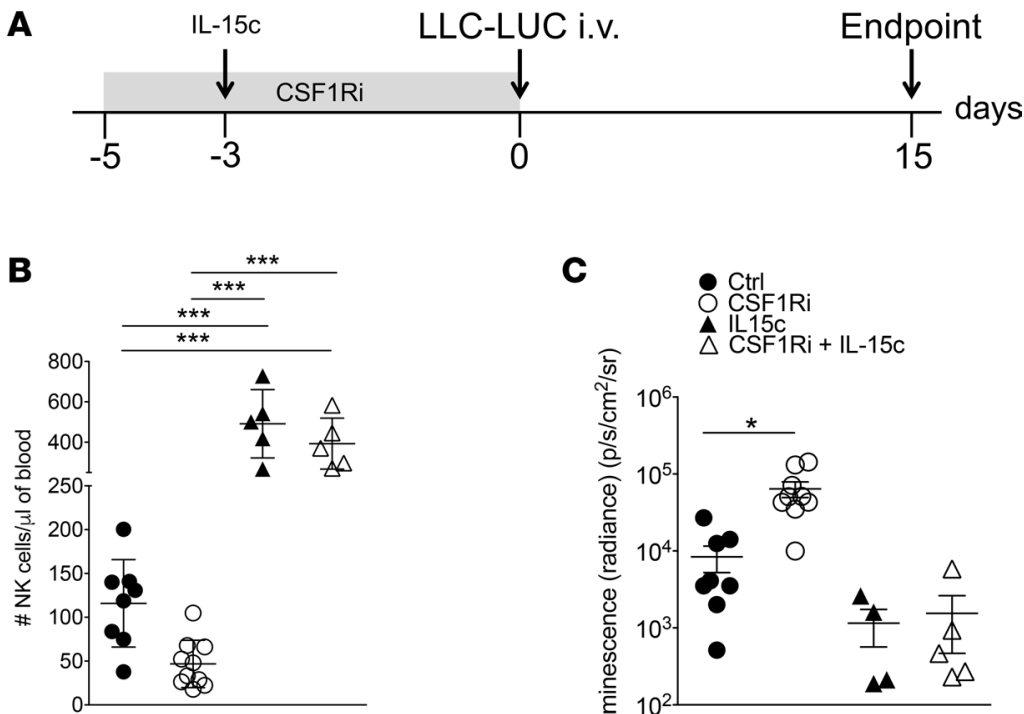

C

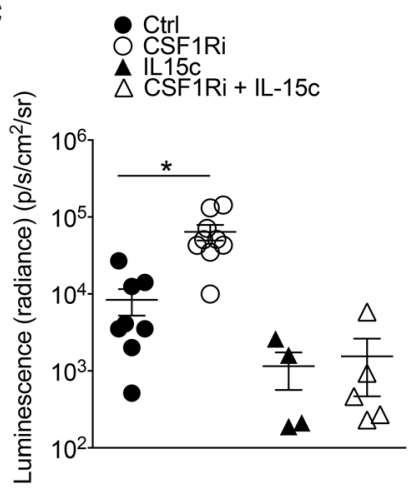

Figure 4. Exogenous IL-15 prevents CSF1Ri-induced loss of NK cells and metastasis. (A) Experimental timeline. (B) Number of circulating NK cells defined as CD45.2+CD3$\mathrm{NK} 1.1^{+} \mathrm{NKp} 46^{+}$live singlets on day 3 . Each symbol represents an individual mouse. Ctrl, $n=9$; CSF1Ri, $n=10$; IL-15c, $n=5$; CSF1Ri + IL-15c, $n=5$. Mean \pm SD. ${ }^{* * *} P<0.005$ (1-way ANOVA with Bonferroni's correction). A representative experiment of 2 is shown. (C) Quantification of tumor burden in the lungs by bioluminescence. Each symbol represents an individual mouse. Ctrl, $n=9$; CSF1Ri, $n=10$; IL-15C, $n=5$; CSF1Ri + IL-15c, $n=5$. Mean \pm SEM. ${ }^{*} P<0.05$ (1-way ANOVA with Bonferroni's correction). A representative experiment of 2 is shown. (D) Representative bioluminescence images of resected lungs at the endpoint.

\section{D}
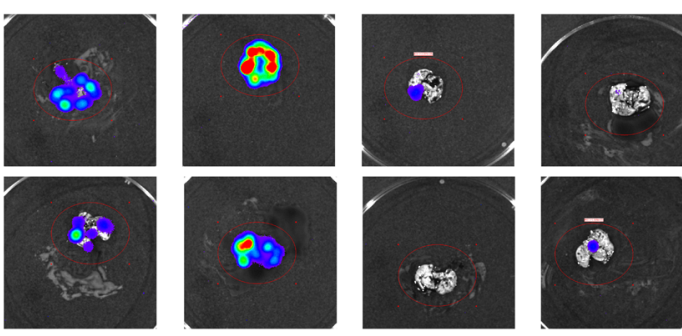

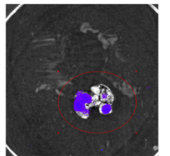

Ctrl
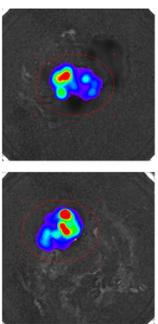

CSF1Ri
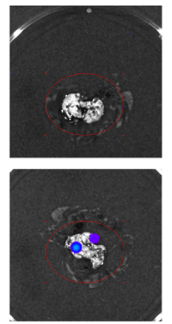

IL-15

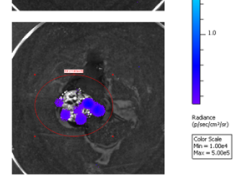

CSF1Ri

29), which restores the number of NK cells in the face of low numbers of CSF1R-dependent myeloid cells. Transpresented IL-15 is also required for survival of $\mathrm{CD}^{+}$memory T cells $(24,45)$. In fact, our observation that CSF1R blockade resulted in a paucity of both $\mathrm{NK}$ and $\mathrm{CD} 8^{+} \mathrm{T}$ cells supports our explanation that loss of IL-15-transpresenting myeloid cells is indirectly responsible for increased metastasis. Although CSF1Ri reduced $\mathrm{CD}^{+} \mathrm{T}$ cell counts, we do not think that $\mathrm{CD}^{+} \mathrm{T}$ cells play a major role in protection against metastatic seeding and progression, as depletion of $\mathrm{CD}^{+} \mathrm{T}$ cells had no impact on these parameters in the experimental systems investigated here.

Together, these data show that NK cells are essential for protection against metastatic seeding and, at the same time, challenge the notion of a major role for myeloid cells other than supporting NK cell survival. This may seem to contradict the recently published findings by Hanna et al., who showed that control of metastasis required the presence of $\mathrm{NR} 4 \mathrm{~A} 1^{+}$patrolling monocytes (46). NR4A1 $1^{+}$patrolling monocytes are Ly $6 \mathrm{C}^{\text {lo }}$ monocytes (47) that are enriched in the microvasculature of the lung. Although this study showed that $\mathrm{NR} 4 \mathrm{~A}^{+}$patrolling monocytes attract NK cells, a functional role for NK cells in protection against metastasis was not addressed. We did not specifically investigate NR4A $1^{+}$monocytes here but expect that CSF1Ri will target NR4A1+ monocytes, as well, because of their expression of CSF1R (46). Furthermore, and as discussed above, the fact that supplementation with IL-15c prevents CSF1Ri-induced metastasis clearly identifies NK cells as the major cell type protecting against metastasis to the lung.

The importance of NK cells in controlling metastasis after i.v. injection of tumor cells has been documented $(2,48-50)$, which suggests that NK cells protect against metastatic seeding by controlling circulating tumor cells. Circulating tumor cells may be particularly vulnerable to NK cells, as oxidative or shear stress can induce increased expression of ligands for NKG2D on tumor cells $(51,52)$. We did not 


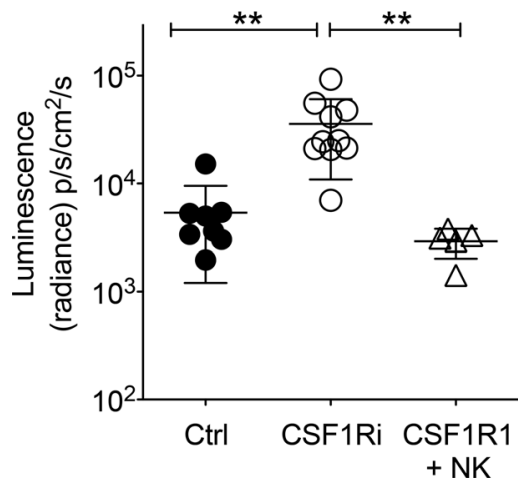

Figure 5. Adoptive transfer of NK cells reverts CSF1Ri-induced metastasis. Three groups of C57BL/6 were treated essentially as described in Figure 4A. Group 1 received daily vehicle starting at day -5 and LLC-LUC on day 0; group 2 received daily CSF1Ri starting at day -5 and LLC-LUC on day 0; group 3 received daily CSF1Ri starting at day -5 and LLC-LUC, as well as $1 \times 10^{6}$ sorted naive NK cells, on day 0 . Metastatic load in the lungs was quantified by bioluminescence on resected lungs on day 15 . Each symbol represents an individual mouse. Ctrl, $n=8$; CSF1Ri, $n=10$; CSF1Ri + NK, $n=5$. ${ }^{* *} P<0.01$ (1-way ANOVA with Bonferroni's correction). A representative experiment of 2 is shown.

investigate here which effector mechanisms NK cells employ to protect against metastatic seeding because there is a consensus in the literature that the dominant effector mechanism NK cells use to protect against metastasis depends on the experimental setting (53-56).

Our findings that neo-adjuvant CSF1Ri promotes metastasis is of clinical relevance because of ongoing clinical studies on CSF1R-targeting therapies. Ries et al. showed that anti-CSF1R treatment induced a clinical response in patients with nonmetastasizing diffuse-type giant cell tumors, a tumor that produces large amounts of macrophage CSF (M-CSF) (14), but clinical data on CSF1R blockade and metastasis are not yet available. However, it has been shown that NK cells protect against metastatic seeding of human cancer cell lines in immunodeficient mice (57) and that homeostasis of human NK cells requires transpresentation of IL-15 by myeloid cells (58). Thus, the available evidence strongly suggests that the crosstalk between NK and CSF1R ${ }^{+}$myeloid cells, the dependence of NK cells on transpresented IL-15, and the control of metastatic seeding follow similar mechanisms in mice and humans.

It should be emphasized, however, that — in our experiments - CSF1Ri-induced metastasis only occurred in a neo-adjuvant setting. Moreover, we have used CSF1Ri as a monotherapy, not precluding the beneficial results of combining CSF1Ri with other immunotherapeutics. Thus, although blockade of CSF1R signaling depletes myeloid cells including TAMS, and may consequently impede tumor progression, it can also promote metastasis when used as neo-adjuvant treatment. This relates to CSF1Ri as neo-adjuvant treatment but presumably also to situations in which micrometastases or metastases are present. Taken together, our results suggest that treatment with CSF1Ri or CSF1R-blocking antibodies bears the risk of increasing metastatic disease in particular conditions, which can be efficiently counteracted by concomitant administration of an NK cell survival factor such as IL-15 (Figure 6).

\section{Methods}

Mice. C57BL/6JOlaHsd1 mice were obtained from Harlan Laboratories (Envigo) and BALB/cJRj mice from Janvier. Il15rat/fl (59), CMV-Cre (60), and MacGreen (61) mice were obtained from the Jackson Laboratory. Il15rallfl and CMV-Cre mice were interbred to obtain Il15ra-deficient mice (referred to here as $I 115 \mathrm{ra}^{-/-}$). Mice were kept under specific pathogen-free conditions at the Laboratory Animal Services Center at the University of Zurich or ETH Phenomics Center at the Swiss Federal Institute of Technology Zurich (Zurich, Switzerland). Six- to 8-week-old female mice were used for all experiments.

Cell lines. LLC1 cells were obtained from ATCC. 4T1 cells were a gift from Michael Detmar (Swiss Federal Institute of Technology in Zurich). LLC and 4T1 cells were cultured in DMEM supplemented with 10\% FBS, 2 mM L-glutamine, penicillin, and streptomycin or RPMI 1640 Medium supplemented with 10\% FBS, 2 mM L-glutamine, penicillin, and streptomycin (all Invitrogen), respectively. LLC cells were lentivirally transduced to express firefly LUC-generating LLC-LUC cells; 4T1 cells were lentivirally transduced to express LUC. Viral particles were a gift from Christian Münz (University of Zurich). Cell lines were confirmed to be free of Mycoplasma and various viruses by Charles River Research Animal Diagnostic Services.

Models of metastasis. For spontaneous metastasis from LLC-LUC tumors, C57BL/6 mice were injected s.c. with $2 \times 10^{5}$ LLC-LUC cells in $100 \mu 1$ PBS. After 25-28 days, mice were anesthetized with $2.5 \%$ Attane (Isoflurane, Piramal Healthcare Ltd.) and $0.04 \mathrm{mg} / \mathrm{kg}$ fentanyl (Kantonsapotheke Zurich) injected i.p. Primary tumors were subsequently resected, and wounds were clipped with Autoclip wound clips 
Untreated
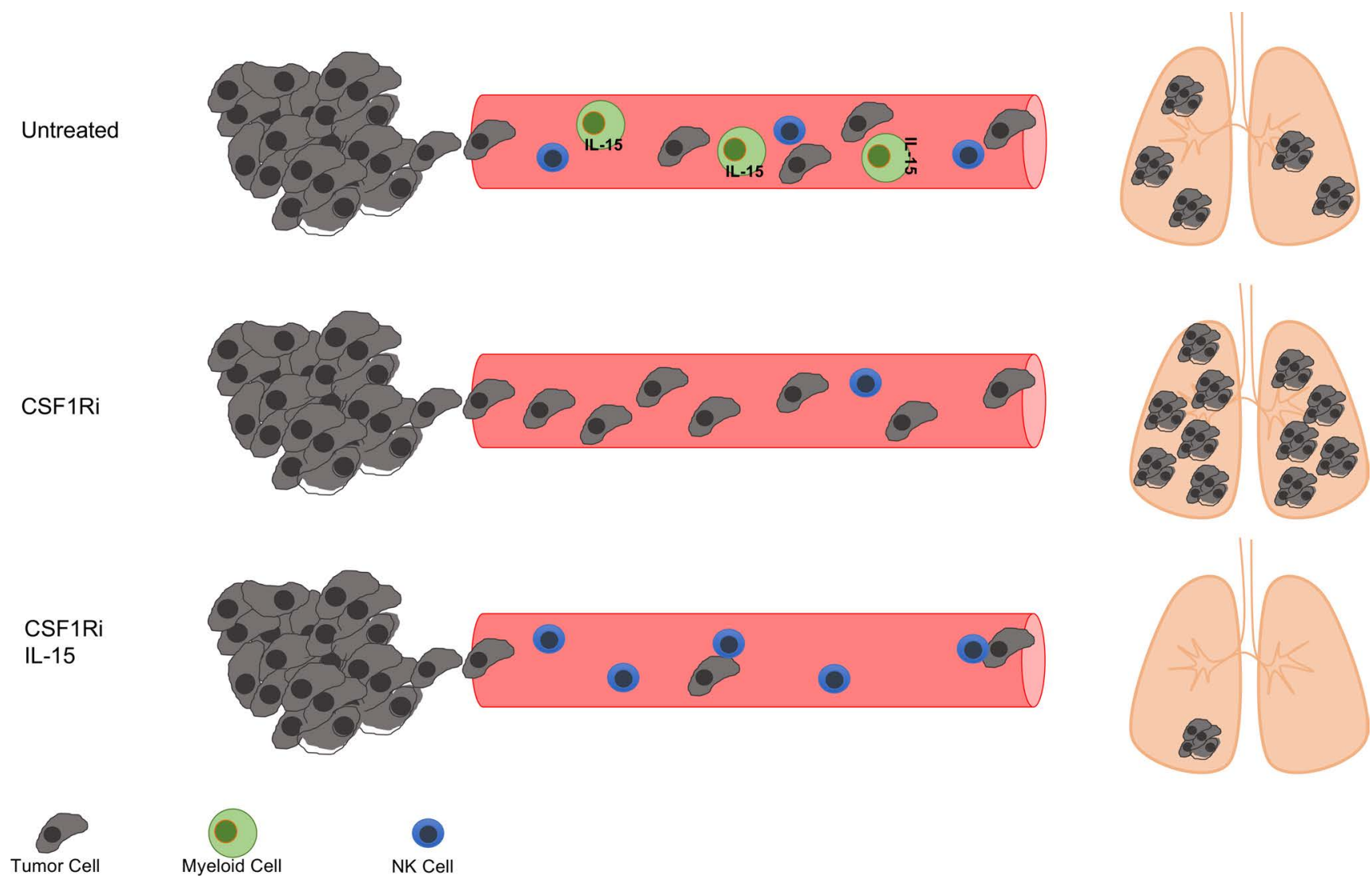

NK Cell

Figure 6. NK cells control metastatic seeding. Blockade of CSF1R results in loss of CSF1R-dependent myeloid cells and, consequently, of transpresented IL-15. This situation induces a paucity of NK cells and promotes metastasis. Administration of exogenous IL-15 to CSF1Ri-treated mice rescues NK cell numbers and reverses increased metastasis in the face of low numbers of myeloid cells.

(BD Biosciences). For postoperative analgesia, Temgesic (Buprenorphine, Schering-Plough) was given s.c. at $0.1 \mathrm{mg} / \mathrm{kg}$ immediately after surgery and in drinking water at $10 \mu \mathrm{g} / \mathrm{ml}$ for 48 hours ad libitum. Three weeks after surgery, mice were anesthetized with $2.5 \%$ Attane and injected i.p. with $150 \mathrm{mg} / \mathrm{kg}$ D-luciferin (TBD-Biodiscovery). In our experience, s.c. LLC-LUC tumors typically give rise to metastasis to lungs, liver, and/or tumor-draining lymph nodes in $50 \%-70 \%$ of the mice. The affected organs contain 1-3 metastatic foci on average. For this reason, we determined the cumulative photon flux of dissected lungs, liver, and tumor-draining lymph nodes using an IVIS 200 imaging system (PerkinElmer). The data are presented as sum of luminescent signals from lung, liver, and 2 tumor-draining lymph nodes for each mouse. Mice with primary tumors below $200 \mathrm{mg}$ at resection were not further analyzed for metastasis development. When interventions started after resection, mice were randomized based on the weight of the primary tumor before start of interventions.

For spontaneous metastasis from orthotopic breast cancer, $1 \times 10^{5} 4 \mathrm{~T} 1$ cells in $50 \mu 1$ PBS were injected into the second mammary fat pad of BALB/c mice, and mice were sacrificed on day 23 or 25 , as indicated. For counting metastases, India ink (Pelikan, $15 \%$ in PBS) was injected intratracheally and lungs were removed, washed with PBS, and fixed in Fekete's solution (62\% ethanol, 3.3\% formaldehyde, $0.25 \mathrm{M}$ acetic acid). Metastatic foci were counted in a blinded fashion using a dissecting microscope. Because $4 \mathrm{~T} 1$ tumors induce metastasis with faster kinetics than LLC-LUC tumors, the size of the primary tumors stayed below the legal limit until the endpoint; therefore, we did not perform surgery in the 4T1 model.

For experimental metastasis, C57BL/ 6 mice were injected i.v. into the tail vein with $5 \times 10^{5}$ LLC-LUC cells in $200 \mu 1$ PBS. Mice were sacrificed on days mentioned in legends of Figures 3, 4, and 5 and Supplemental Figures 8 and 9; bioluminescence was measured in dissected lungs as described above.

Treatment of mice. To deplete $\mathrm{CD} 8^{+} \mathrm{T}$ cells, $\mathrm{CD}^{+} \mathrm{T}$ cells, or NK cells, mice were injected with $500 \mu \mathrm{g}$ anti-CD8 (clone YTS169.4, hybridoma originally obtained from H. Waldmann, Oxford, United Kingdom), $500 \mu \mathrm{g}$ anti-CD4 (clone GK1.5, ATCC), or $200 \mu \mathrm{g}$ anti-NK1.1 (clone PK136, ATCC), respectively. A single 
injection of the respective antibody resulted in depletion of $>90 \%$ of $\mathrm{CD}^{+}$or $\mathrm{CD} 8^{+} \mathrm{T}$ cells for at least 18 days, as determined by flow cytometry (data not shown for CD4). Antibodies were injected as depicted in the experimental timelines. Anti-CD8, anti-CD4, and anti-NK1.1 antibodies were purified from hybridoma culture supernatant using protein $\mathrm{G}$ sepharose 4 Fast Flow (MilliporeSigma). Anti-CSF1R antibody (clone AFS98) was obtained from BioXCell and was used at $500 \mu \mathrm{g}$ per dose. All antibodies were administered i.p. in $200 \mu \mathrm{l}$ PBS. Full depletion of the targeted population was confirmed by flow cytometry on blood 2 days after injection of the antibody in every experiment.

CSF1Ri BLZ945 was supplied by Novartis Institutes for Biomedical Research (Emeryville, California, USA) and dissolved at $12.5 \mathrm{mg} / \mathrm{ml}$ in $20 \%$ Captisol as vehicle. CSF1Ri or vehicle were administered daily per os (p.o.) at $200 \mathrm{mg} / \mathrm{kg}$.

IL-15 complexes were prepared by mixing recombinant murine IL-15 (Affymetrix eBioscience) and recombinant murine IL-15R $\alpha$ (R\&D Biosystems) in a 1:1 molar ratio and by subsequent incubation at $37^{\circ} \mathrm{C}$ for 30 minutes as described (28). IL-15 complexes were administered i.p. at $4.5 \mu \mathrm{g} / \mathrm{mouse}$ in 200 $\mu 1$ of PBS.

For adoptive transfer of NK cells, live $\mathrm{CD} 45^{+} \mathrm{CD} 3^{-} \mathrm{NK} 1.1^{+}$single cells were sorted using a FACS Melody (BD Biosciences) with a 100- $\mu$ m nozzle from spleens from naive C57BL/6 mice. Sorted NK cells were $>98 \%$ pure (data not shown). At the time point of i.v. tumor cell injection, $1 \times 10^{6} \mathrm{NK}$ cells were i.v. injected with $100 \mu \mathrm{PBS}$

Flow cytometry. Primary tumors were collected in PBS, cut into pieces, and digested for 45 minutes at $37^{\circ} \mathrm{C}$ in RPMI Medium containing $10 \% \mathrm{FBS}, 1 \mathrm{mg} / \mathrm{ml}$ collagenase IV, and $2.6 \mu \mathrm{g} / \mathrm{ml}$ DNase I (both MilliporeSigma). Samples were washed with PBS by centrifugation for 5 minutes at $350 \mathrm{~g}$, and the pellet was resuspended in PBS and filtered to remove debris. For surface staining, antibodies against the following proteins were used: CD3 (clone 17A2), CD4 (clone RM4-5), CD8a (clone 53-6.7), CD11b (clone M1/70), CD19 (clone 6D5), CD27 (clone LG.3A10), CSF1R (clone AFS98), CD45.2 (clone 104), CD49b (clone DX5), CD69 (clone H1.2F3), DNAM-1 (clone 10E5), F4/80 (clone BM8), FasL (clone MFL3), FoxP3 (clone FJK.16s), KLRG1 (clone 2F1), Ly6C (clone HK1.4), Ly6G (clone 1A8), NK1.1 (clone PK136), and NKp46 (clone 29A1.4). For viability staining, Zombie Violet Fixable Viability Kit was used. Anti-FoxP3 (FJK.16s) and FoxP3-staining buffers were purchased from eBioscience; all other antibodies and reagents mentioned above in this paragraph were obtained from BioLegend. Samples were incubated in PBS for 25 minutes at $4^{\circ} \mathrm{C}$. For quantitative analysis, CountBright absolute counting beads were used (Thermo Fisher Scientific). In all staining, dead cells were excluded using Live/Dead fixable staining reagents (Invitrogen), and doublets were excluded by forward scatter A (FSC-A) versus FSC-H and side scatter A (SSC-A) versus SSC-H gating. Samples were acquired using a CyAn ADP 9 flow cytometer (Beckman Coulter) or FACS LSRII Fortessa (BD Biosciences) and analyzed using FlowJo v9.8.5 software (Tree Star Inc.).

IL-15 transpresentation assay. As a measure of IL-15R signaling to NK cells, pSTAT5 was measured (25). NK1.1 $1^{+} \mathrm{CD}^{-}$cells were sorted from the spleen of C57BL/6 mice and were stained after sorting with $10 \mu \mathrm{M}$ Celltracker Violet (Invitrogen) according to the manufacturer's instructions. NK1.1 ${ }^{-} \mathrm{CD}$ -

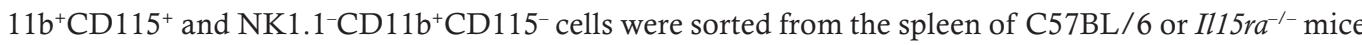
that were injected i.p. 24 hours before with $150 \mu \mathrm{g}$ poly-IC (MilliporeSigma) in PBS. Only live singlets were considered for sorting. Cell sorting was performed with a FACSMelody (BD Biosciences) using a $100-\mu \mathrm{m}$ nozzle. Sorted cells had a purity $>95 \%$. Fifty-thousand NK cells were cultured with an equal number of WT NK1.1-CD $11 \mathrm{~b}^{+} \mathrm{CD} 115^{+}$cells, WT NK1.1-CD $11 \mathrm{~b}^{+} \mathrm{CD} 115^{-}$cells, Il15ra ${ }^{-/-} \mathrm{NK} 1.1^{-} \mathrm{CD}-$ $11 \mathrm{~b}^{+} \mathrm{CD} 115^{+}$cells, Il15ra ${ }^{-/-} \mathrm{NK} 1.1^{-} \mathrm{CD} 11 \mathrm{~b}^{+} \mathrm{CD} 115^{-}$cells, or with medium alone. Cultures were performed in 96-well round-bottom plates in a volume of $150 \mu 1$ medium (RPMI 1640 Medium supplemented with $10 \%$ FBS, 2 mM L-glutamine, penicillin, and streptomycin) for 30 minutes at $37^{\circ} \mathrm{C}$. Cells were subsequently stained for pSTAT5 as described (62). Briefly, cells were collected and fixed with $2 \%$ paraformaldehyde for 20 minutes at $4^{\circ} \mathrm{C}$. Cells were spun down, resuspended in $1 \mathrm{ml}$ ice-cold methanol, and incubated for 20 minutes at $4^{\circ} \mathrm{C}$. Cells were washed twice with $0.5 \%$ FCS in PBS and stained with anti-pSTAT5 (clone 47, BD Biosciences) for 45 minutes at $4^{\circ} \mathrm{C}$. pSTAT5 expression was measured after gating on live, Celltracker Violet ${ }^{+}$NK cells using a CyAn ADP 9 flow cytometer (Beckman Coulter) and FlowJo v9.8.5 software (Tree Star Inc.).

Statistics. For comparison of 2 experimental groups, 2-tailed Student's $t$ test with Welch's correction was performed. More than 2 groups were compared using the 1-way ANOVA test with Bonferroni's 
correction. Frequencies of metastases in the LLC-LUC model were compared with the $\chi^{2}$ test based on the frequencies in the control groups of each experiment. All tests were performed with GraphPad Prism 5.0 and GraphPad QuickCalcs (GraphPad Software). ${ }^{*} P<0.05,{ }^{* *} P<0.01,{ }^{* *} P<0.005$. Unless stated otherwise, data are shown as mean \pm SD.

Study approval. Animal experiments were performed in accordance with the Swiss federal and cantonal regulations on animal protection and were approved by The Cantonal Veterinary Office Zurich (license number 65/2015 to MVDB).

\section{Author contributions}

MB, PTDL, and MVDB conceived experiments; MB, PTDL, MVDB, VC, YM, IO, ST, GL, A. Gupta, A. Gagliardi, MV, and NM performed experiments; MB, PTDL, and MVDB wrote the manuscript; MVDB, PTDL, and BB secured funding; JS and RS provided reagents.

\section{Acknowledgments}

We thank Anne Müller, Stefanie Hiltbrunner, Alexander Knuth, Lubor Borsig, and Christian Münz for valuable input and support. We thank the personnel from the Laboratory Animal Service Center (University of Zurich) and ETH Phenomic Center (Swiss Federal Institute of Technology Zurich) for expert animal care. This work was financially supported by the Swiss National Science Foundation (SNSF), the Swiss Cancer League (Oncosuisse), the Jubiläumsstiftung der Schweizerischen Lebensversicherungs- und Rentenanstalt für Volksgesundheit und medizinische Forschung, the University of Zurich Forschungskredit, and the University Research Priority Program (URPP) Translational Cancer Research.

Address correspondence to: Maries van den Broek, Institute of Experimental Immunology, University of Zurich, Winterthurerstrasse 190, 8057 Zurich, Switzerland. Phone: 41.44.635.3722; Email: vandenbroek@ immunology.uzh.ch.

JS's present address is: IDEAYA San Francisco, South San Francisco, California, USA.

A. Gupta's present address is: Visceral and Transplantation Surgery, University Hospital Zurich, Zurich, Switzerland.

1. Chaffer CL, Weinberg RA. A perspective on cancer cell metastasis. Science. 2011;331(6024):1559-1564.

2. Spiegel A, et al. Neutrophils Suppress Intraluminal NK Cell-Mediated Tumor Cell Clearance and Enhance Extravasation of Disseminated Carcinoma Cells. Cancer Discov. 2016;6(6):630-649.

3. Mlecnik B, et al. The tumor microenvironment and Immunoscore are critical determinants of dissemination to distant metastasis. Sci Transl Med. 2016;8(327):327ra26.

4. Wolchok JD, et al. Nivolumab plus ipilimumab in advanced melanoma. N Engl J Med. 2013;369(2):122-133.

5. Robert C, et al. Ipilimumab plus dacarbazine for previously untreated metastatic melanoma. N Engl J Med. 2011;364(26):2517-2526.

6. Parkhurst MR, Riley JP, Dudley ME, Rosenberg SA. Adoptive transfer of autologous natural killer cells leads to high levels of circulating natural killer cells but does not mediate tumor regression. Clin Cancer Res. 2011;17(19):6287-6297.

7. Michel T, et al. Mouse lung and spleen natural killer cells have phenotypic and functional differences, in part influenced by macrophages. PLoS ONE. 2012;7(12):e51230.

8. Umemura N, et al. Tumor-infiltrating myeloid-derived suppressor cells are pleiotropic-inflamed monocytes/macrophages that bear M1- and M2-type characteristics. J Leukoc Biol. 2008;83(5):1136-1144.

9. Rakhmilevich AL, et al. Tumor-associated myeloid cells can be activated in vitro and in vivo to mediate antitumor effects. Cancer Immunol Immunother. 2012;61(10):1683-1697.

10. Broz ML, et al. Dissecting the tumor myeloid compartment reveals rare activating antigen-presenting cells critical for $\mathrm{T}$ cell immunity. Cancer Cell. 2014;26(5):638-652.

11. Qian BZ, et al. CCL2 recruits inflammatory monocytes to facilitate breast-tumour metastasis. Nature. 2011;475(7355):222-225.

12. Kitamura T, et al. CCL2-induced chemokine cascade promotes breast cancer metastasis by enhancing retention of metastasis-associated macrophages. J Exp Med. 2015;212(7):1043-1059.

13. Qian B, et al. A distinct macrophage population mediates metastatic breast cancer cell extravasation, establishment and growth. PLoS One. 2009;4(8):e6562.

14. Ries $\mathrm{CH}$, et al. Targeting tumor-associated macrophages with anti-CSF-1R antibody reveals a strategy for cancer therapy. Cancer Cell. 2014;25(6):846-859.

15. Zhu Y, et al. CSF1/CSF1R blockade reprograms tumor-infiltrating macrophages and improves response to T-cell checkpoint immunotherapy in pancreatic cancer models. Cancer Res. 2014;74(18):5057-5069.

16. Pyonteck SM, et al. CSF-1R inhibition alters macrophage polarization and blocks glioma progression. Nat Med. 2013;19(10):1264-1272. 
17. Quail DF, et al. The tumor microenvironment underlies acquired resistance to CSF-1R inhibition in gliomas. Science. 2016;352(6288):aad3018.

18. Cassier PA, et al. CSF1R inhibition with emactuzumab in locally advanced diffuse-type tenosynovial giant cell tumours of the soft tissue: a dose-escalation and dose-expansion phase 1 study. Lancet Oncol. 2015;16(8):949-956.

19. Cannarile MA, Weisser M, Jacob W, Jegg AM, Ries CH, Rüttinger D. Colony-stimulating factor 1 receptor (CSF1R) inhibitors in cancer therapy. J Immunother Cancer. 2017;5(1):53.

20. Cecchini MG, et al. Role of colony stimulating factor-1 in the establishment and regulation of tissue macrophages during postnatal development of the mouse. Development. 1994;120(6):1357-1372.

21. Kumar V, et al. Cancer-Associated Fibroblasts Neutralize the Anti-tumor Effect of CSF1 Receptor Blockade by Inducing PMN-MDSC Infiltration of Tumors. Cancer Cell. 2017;32(5):654-668.e5.

22. Stott SL, et al. Isolation and characterization of circulating tumor cells from patients with localized and metastatic prostate cancer. Sci Transl Med. 2010;2(25):25ra23.

23. Soderquest K, et al. Monocytes control natural killer cell differentiation to effector phenotypes. Blood. 2011;117(17):4511-4518.

24. Burkett PR, Koka R, Chien M, Chai S, Boone DL, Ma A. Coordinate expression and trans presentation of interleukin (IL)-15Ralpha and IL-15 supports natural killer cell and memory CD8+ T cell homeostasis. J Exp Med. 2004;200(7):825-834.

25. Lee GA, Liou YH, Wang SW, Ko KL, Jiang ST, Liao NS. Different NK cell developmental events require different levels of IL-15 trans-presentation. J Immunol. 2011;187(3):1212-1221.

26. Dubois S, Mariner J, Waldmann TA, Tagaya Y. IL-15Ralpha recycles and presents IL-15 In trans to neighboring cells. Immunity. 2002;17(5):537-547.

27. Mortier E, Woo T, Advincula R, Gozalo S, Ma A. IL-15Ralpha chaperones IL-15 to stable dendritic cell membrane complexes that activate NK cells via trans presentation. J Exp Med. 2008;205(5):1213-1225.

28. Elpek KG, Rubinstein MP, Bellemare-Pelletier A, Goldrath AW, Turley SJ. Mature natural killer cells with phenotypic and functional alterations accumulate upon sustained stimulation with IL-15/IL-15Ralpha complexes. Proc Natl Acad Sci USA. 2010;107(50):21647-21652.

29. Stoklasek TA, Schluns KS, Lefrançois L. Combined IL-15/IL-15Ralpha immunotherapy maximizes IL-15 activity in vivo. J Immunol. 2006;177(9):6072-6080.

30. Malaisé M, et al. KLRG1+ NK cells protect T-bet-deficient mice from pulmonary metastatic colorectal carcinoma. J Immunol. 2014;192(4):1954-1961.

31. Hayakawa Y, Smyth MJ. CD27 dissects mature NK cells into two subsets with distinct responsiveness and migratory capacity. J Immunol. 2006;176(3):1517-1524.

32. Qian BZ, Pollard JW. Macrophage diversity enhances tumor progression and metastasis. Cell. 2010;141(1):39-51.

33. Strauss L, et al. RORC1 Regulates Tumor-Promoting "Emergency" Granulo-Monocytopoiesis. Cancer Cell. 2015;28(2):253-269.

34. Van Overmeire E, et al. M-CSF and GM-CSF Receptor Signaling Differentially Regulate Monocyte Maturation and Macrophage Polarization in the Tumor Microenvironment. Cancer Res. 2016;76(1):35-42.

35. Hume DA, MacDonald KP. Therapeutic applications of macrophage colony-stimulating factor-1 (CSF-1) and antagonists of CSF-1 receptor (CSF-1R) signaling. Blood. 2012;119(8):1810-1820.

36. Escamilla J, et al. CSF1 receptor targeting in prostate cancer reverses macrophage-mediated resistance to androgen blockade therapy. Cancer Res. 2015;75(6):950-962.

37. Moughon DL, et al. Macrophage Blockade Using CSF1R Inhibitors Reverses the Vascular Leakage Underlying Malignant Ascites in Late-Stage Epithelial Ovarian Cancer. Cancer Res. 2015;75(22):4742-4752.

38. Strachan DC, et al. CSF1R inhibition delays cervical and mammary tumor growth in murine models by attenuating the turnover of tumor-associated macrophages and enhancing infiltration by CD8+ T cells. Oncoimmunology. 2013;2(12):e26968

39. Mao Y, Eissler N, Blanc KL, Johnsen JI, Kogner P, Kiessling R. Targeting Suppressive Myeloid Cells Potentiates Checkpoint Inhibitors to Control Spontaneous Neuroblastoma. Clin Cancer Res. 2016;22(15):3849-3859.

40. Swierczak A, et al. The promotion of breast cancer metastasis caused by inhibition of CSF-1R/CSF-1 signaling is blocked by targeting the G-CSF receptor. Cancer Immunol Res. 2014;2(8):765-776.

41. Kowanetz M, et al. Granulocyte-colony stimulating factor promotes lung metastasis through mobilization of Ly6G+Ly6C+ granulocytes. Proc Natl Acad Sci USA. 2010;107(50):21248-21255.

42. Coffelt SB, et al. IL-17-producing $\gamma \delta \mathrm{T}$ cells and neutrophils conspire to promote breast cancer metastasis. Nature. 2015;522(7556):345-348.

43. Bonapace L, et al. Cessation of CCL2 inhibition accelerates breast cancer metastasis by promoting angiogenesis. Nature. 2014;515(7525):130-133.

44. Ranson T, Vosshenrich CA, Corcuff E, Richard O, Müller W, Di Santo JP. IL-15 is an essential mediator of peripheral NK-cell homeostasis. Blood. 2003;101(12):4887-4893.

45. Sato N, Patel HJ, Waldmann TA, Tagaya Y. The IL-15/IL-15Ralpha on cell surfaces enables sustained IL-15 activity and contributes to the long survival of CD8 memory T cells. Proc Natl Acad Sci USA. 2007;104(2):588-593.

46. Hanna RN, et al. Patrolling monocytes control tumor metastasis to the lung. Science. 2015;350(6263):985-990.

47. Carlin LM, et al. Nr4a1-dependent Ly6C(low) monocytes monitor endothelial cells and orchestrate their disposal. Cell. 2013;153(2):362-375.

48. Vermijlen D, et al. Hepatic natural killer cells exclusively kill splenic/blood natural killer-resistant tumor cells by the perforin/ granzyme pathway. J Leukoc Biol. 2002;72(4):668-676.

49. Paolino M, et al. The E3 ligase Cbl-b and TAM receptors regulate cancer metastasis via natural killer cells. Nature. 2014;507(7493):508-512.

50. Hanna N, Fidler IJ. Role of natural killer cells in the destruction of circulating tumor emboli. J Natl Cancer Inst. 1980;65(4):801-809.

51. Yamamoto K, Fujiyama Y, Andoh A, Bamba T, Okabe H. Oxidative stress increases MICA and MICB gene expression in the human colon carcinoma cell line (CaCo-2). Biochim Biophys Acta. 2001;1526(1):10-12.

52. Piskounova E, et al. Oxidative stress inhibits distant metastasis by human melanoma cells. Nature. 2015;527(7577):186-191

53. Chan CJ, et al. The receptors CD96 and CD226 oppose each other in the regulation of natural killer cell functions. Nat Immunol. 
2014;15(5):431-438.

54. Smyth MJ, et al. Perforin is a major contributor to NK cell control of tumor metastasis. J Immunol. 1999;162(11):6658-6662.

55. van den Broek MF, Kägi D, Zinkernagel RM, Hengartner H. Perforin dependence of natural killer cell-mediated tumor control in vivo. Eur J Immunol. 1995;25(12):3514-3516.

56. Wallin RP, Screpanti V, Michaëlsson J, Grandien A, Ljunggren HG. Regulation of perforin-independent NK cell-mediated cytotoxicity. Eur J Immunol. 2003;33(10):2727-2735.

57. Le Dévédec SE, et al. An improved model to study tumor cell autonomous metastasis programs using MTLn3 cells and the Rag2(-/-) gammac (-/-) mouse. Clin Exp Metastasis. 2009;26(7):673-684.

58. Huntington ND, et al. IL-15 trans-presentation promotes human NK cell development and differentiation in vivo. J Exp Med. 2009;206(1):25-34.

59. Mortier E, et al. Macrophage- and dendritic-cell-derived interleukin-15 receptor alpha supports homeostasis of distinct CD8+ T cell subsets. Immunity. 2009;31(5):811-822.

60. Schwenk F, Baron U, Rajewsky K. A cre-transgenic mouse strain for the ubiquitous deletion of loxP-flanked gene segments including deletion in germ cells. Nucleic Acids Res. 1995;23(24):5080-5081.

61. Sasmono RT, Williams E. Generation and characterization of MacGreen mice, the Cfs1r-EGFP transgenic mice. Methods Mol Biol. 2012;844:157-176

62. Croxford AL, et al. The Cytokine GM-CSF Drives the Inflammatory Signature of CCR2+ Monocytes and Licenses Autoimmunity. Immunity. 2015;43(3):502-514. 